\title{
Correspondence Glutamate Changes in Anterior Cingulate Cortex Following CCK-4 Infusion
}

\author{
Richard J Maddock*,I \\ IImaging Research Center, Department of Psychiatry, University of California Davis, Sacramento, CA, USA
}

Neuropsychopharmacology (2014) 39, 2706; doi:I0.1038/npp.2014.93; published online I8 June 20 I4

Zwanzger et al (2013) recent article entitled 'Acute shift in glutamate concentrations following experimentally induced panic with cholecystokinin tetrapeptide-a 3T-MRS study in healthy subjects' demonstrates a creative experimental design for studying the neurochemical processes underlying panic attacks. In 18 healthy volunteers, they acquired a series of $1 \mathrm{H}-\mathrm{MRS}$ measurements in the anterior cingulate cortex to measure the Glx signal (arising primarily from glutamate) before and after an intravenous injection of a panic-inducing dose of CCK-4. They concluded that 'CCK-4-induced panic was accompanied by a rapid and significant increase of Glx concentrations in the ACC.' Unfortunately, they used a statistically invalid approach to test the likelihood that their observations could have occurred by chance.

Their experimental design included six 5-minute MRS acquisitions, each yielding a measurement of Glx. The first measurement was made before the CCK-4 injection and was considered the baseline measurement. The five subsequent Glx measurements were considered post CCK-4 measurements. To test their hypothesis that CCK-4 causes an increase in ACC Glx, the authors used a repeated measures analysis of variance to compare the baseline Glx value to the maximum Glx value observed in any of the five post CCK-4 measurements. They reported a highly significant result: $\mathrm{F}(1,17)=15.94, P=0.001$. However, this statistical approach implicitly assumes that the null hypothesis is zero, or 'no difference' between the baseline and the maximum subsequent Glx value. This is clearly incorrect. In a series of six random numbers, the first number will be lower than the maximum of the subsequent five numbers $83 \%$ of the time. To illustrate the seriousness of this statistical illusion, I used a random number generator to create three data sets similar to that reported by Zwanzger et al (2013). Each data set included 18 'subjects' and each subject was represented by a series of six random numbers. The random numbers were generated to have a mean and standard deviation identical to those inferred from Figure 2 in the Zwanzger et al (2013 article. Running a repeated measures ANOVA comparing 'baseline' to 'Maximum post baseline' gave the following results for the three randomly generated data sets: $\mathrm{F}(1,17)=$ $34.67, F(1,17)=21.85$, and $F(1,17)=17.55$-all 'highly significant' if one assumes the null hypothesis is zero difference. Yet, the F ratio reported by Zwanzger et al (2013) was lower than any of the $\mathrm{F}$ ratios I obtained using random numbers as 'data.' The same statistical illusion invalidates their analysis of the heart rate change following CCK-4.

The article by Zwanzger et al tests an interesting hypothesis and includes a scholarly and valuable discussion of the potential role of glutamatergic mechanisms in panic disorder. Unfortunately, the empirical data presented do not support the conclusion that CCK-4 induces a rise in ACC Glx. In fact, the pattern of changes in Glx they observed following CCK-4 resembles what one would expect to see by chance.

\section{FUNDING AND DISCLOSURE}

We declare that, except for income received from our primary employer, no financial support or compensation has been received from any individual or corporate entity over the past 3 years for research or professional service and there are no personal financial holdings that could be perceived as constituting a potential conflict of interest. The authors declare no conflict of interest.

\section{REFERENCE}

Zwanzger P, Zavorotnyy M, Gencheva E, Diemer J, Kugel H, Heindel W et al (2013). Acute shift in glutamate concentrations following experimentally induced panic with cholecystokinin tetrapeptide-a 3T-MRS study in healthy subjects. Neuropsychopharmacology 38: 1648-1654.

*Correspondence: Professor RJ Maddock, Imaging Research Center, Department of Psychiatry, University of California Davis, $4701 \times$ Street, Sacramento, CA 95817, USA, Tel: 916734 3286, Fax: 9167348750 ,

E-mail: rjmaddock@ucdavis.edu 